www.nature.com/clinicalpractice/onc

\title{
Drug Insight: gastrointestinal and hepatic adverse effects of molecular- targeted agents in cancer therapy
}

Yohann Loriot, Gabriel Perlemuter, David Malka, Frédérique Penault-Llorca, Valérie Boige, Eric Deutsch, Christophe Massard, Jean Pierre Armand and Jean-Charles Soria

Nature Clinical Practice Oncology (2008) 5: 268-278 doi:10.1038/ncponc1087

We regret that the above article, printed in the May 2008 issue of the journal, has an error in the name of the fourth author. The name is printed correctly above. 